\title{
Adenoma de hipófise em uma gata com hiperadrenocorticismo
}

\author{
Pituitary adenoma in a cat with hyperadrenocorticism \\ Katia Barão Corgozinho', Cristiane Belchior ${ }^{2}$, Raquel Calixto de Souza ${ }^{3}$, \\ Juliana da Silva Leite ${ }^{4}$ \& Ana Maria Reis Ferreira ${ }^{4}$
}

\begin{abstract}
Background: Feline Cushing's syndrome (FCS) is a disorder of excessive cortisol secretion by the adrenal glands and is rare in cats. The most frequently observed clinical signs are polyuria, polydipsia, and polyphagia which are also consistent with diabetes mellitus. These diabetic cats are often insulin resistants. The dexamethasone suppression test is considered the test of choice for the diagnosis of hyperadrenocorticism. The majority of cats with naturally occurring Cushing's syndrome have pituitary-dependent hyperadrenocorticism and it is caused by functional microadenoma or macroadenoma pituitary. Computed tomography or magnetic resonance imaging is helpful in diagnosis of pituitary tumors. Treatments of pituitary-dependent hyperadrenocorticism include surgery of the pituitary or adrenals, radiation of the pituitary, and medical therapies. Bilateral adrenalectomy continues to represent the best long-term therapeutic strategy until hypophysectomy becomes more widely available. This paper reports a cat with macroadenoma pituitary causing hiperadrenocorticism and insulin resistance.

Case: A 12-year-old female castrated Brazilian shorthair cat was referred to the veterinary due to polyuria, polydipsia, weight loss and polyphagia. The presence of hyperglycemia (blood glucose $>250 \mathrm{mg} / \mathrm{dl}$ ), glucosuria and elevated fructosamine concentration revealed diabetes mellitus. Insulin therapy was introduced but the glycemia was poorly controlled despite the high dose of insulin. Concomitant disease was suspected. Abdominal ultrasonography revealed bilaterally enlarged adrenals. The dexamethasone suppression test showed pituitary-dependent hyperadrenocorticism. Computed tomography or hypophysectomy wasn't available. Medical therapy with mitotane was introduced but anorexia and vomiting developed. Bilateral adrenalectomy was performed without complications and histological evaluation of adrenal revealed hyperplasia. After surgery, treatment with mineralcorticoids and glucocorticoids was introduced. The cat had resolution of clinical signs and insulin requirements were decreased. According to the owner, three weeks after surgery, the cat showed abnormal behavior, compulsive walking and circling. The cat died eight months after bilateral adrenalectomy. A complete necropsy was performed and histopathological examination confirmed the pituitary macroadenoma.

Discussion: Insulin resistance should be suspected in diabetic cat if control of glycemia is poor despite the high insulin dosage. Clinical signs related to poorly controlled diabetes mellitus are common in cats with hyperadrenocorticism. Hyperadrenocorticism can cause severe insulin resistance and it is often associated with a pituitary macrotumor. Pituitary tumors may lead to hypercotisolism. Bilateral adrenalectomy is a viable alternative to transphenoidal hypophysectomy for treatment of feline pituitary-dependent hyperadrenocorticism when hypophysectomy is not available. Neurological signs can be a result of pituitary tumors and they can get worse after the adrenalectomy because of the enlargement of the tumor. Despite of clinical signs, the cat had improved in response to the bilateral adrenalectomy and had a good quality of life during eight months after surgery.
\end{abstract}

Keywords: pituitary, hyperadrenocorticism, cat.

Descritores: hipófise, hiperadrenocorticismo, gato. 


\section{INTRODUÇÃO}

As desordens da glândula pituitária são raras em gatos e geralmente relacionadas à neoplasia da pars distalis ou pars intermedia, mais comumente adenoma [2]. A maioria dos tumores pituitários é afuncional e geralmente achado de necropsia. Os sinais clínicos observados são disfunção do sistema nervoso central e hipopituitarismo relacionados à compressão tecidual pela neoplasia [2]. Quando a neoplasia é funcional, desordens clínicas são relacionadas à produção excessiva do hormônio adrenocorticotrófico (ACTH) ou hormônio do crescimento $(\mathrm{GH})$, levando ao hiperadrenocorticismo ou acromegalia, respectivamente $[2,4,5]$.

Esse trabalho tem como objetivo relatar uma gata com hiperadrenocorticismo secundário a adenoma pituitário.

\section{RELATO DE CASO}

Gata com 12 anos de idade, sem raça definida, foi atendida por causa da ingestão excessiva de água e grande volume urinário. A glicemia, a urinálise e a dosagem de frutosamina sérica revelaram diabetes mellitus. A insulinoterapia foi iniciada utilizando a insulina glargina ${ }^{1}(0,5 \mathrm{UI} / \mathrm{kg}$ BID SC) e mesmo com altas doses não se obteve o controle glicêmico. $\mathrm{O}$ abdômen abaulado e o não controle glicêmico indicaram a presença de doença concomitante, suspeitando-se de hiperadrenocorticismo. A ultrasonografia revelou aumento das duas glândulas adrenais, a esquerda medindo $1,38 \times 0,6$ centímetros e a direita 1,25 x 0,68 centímetros, pâncreas aumentado de volume e menos ecogênico e fígado aumentado de volume com ecogenicidade aumentada.

A pesquisa para hiperadrenocorticismo, através do teste de supressão com dexametasona ${ }^{2}(0,1$ $\mathrm{mg} / \mathrm{kg}$ IV dose única) revelou hiperadrenocorticismo. A dosagem de aldosterona revelou aumento exacerbado desse hormônio, mas os exames bioquímicos da gata não demonstraram hipernatremia ou hipocaliemia. Um diagnóstico de hiperadrenocorticismo hipófise-dependente foi suspeitado após o teste de supressão por dexametasona e avaliação ultrasonográfica (acometimento bilateral). Como não se disponibilizava de tomografia computadorizada na veterinária e nem a possibilidade de hipofisectomia, a terapia clínica utilizando mitotane ${ }^{3}(25 \mathrm{mg} / \mathrm{kg}$ BID PO) foi iniciada, porém a gata apresentou anorexia e vômito, sendo suspensa a medicação. Então, optouse pelo tratamento cirúrgico através da adrenalectomia bilateral (Figura 1 A, B e C). Durante a cirurgia, foi aplicada dexametasona $(0,2 \mathrm{mg} / \mathrm{kg} \mathrm{IV})$ após a retirada da primeira glândula adrenal e repetida após a cirurgia por via intramuscular.

Após a cirurgia, foi suplementado mineralocorticóide usando fludrocortisona ${ }^{4}(0,1 \mathrm{mg} /$ gato SID $\mathrm{PO})$ e corticóide à base de prednisolona ${ }^{5}(1,25 \mathrm{mg} /$ gato SID PO). O exame histopatológico das glândulas adrenais revelou hiperplasia bilateral moderada da zona fasciculada. Nas duas semanas seguintes à cirurgia, a gata estava ótima, comendo e com a glicemia controlada. Apresentava melhora dos sinais clínicos e redução na dose de insulina. Porém, na terceira semana pós-cirúrgica, a gata começou a andar compulsivamente e em círculo. Após oito meses, a gata veio a óbito. A necropsia revelou uma hipófise aumentada de tamanho (Figura 1 D), medindo um centímetro de diâmetro e a histopatologia confirmou macroadenoma de hipófise.

\section{DISCUSSÃO}

Quando um paciente diabético não obtém o controle glicêmico mesmo recebendo altas doses de insulina, hiperadrenocorticismo deve ser suspeitado [5]. Em nosso relato, a suspeita do não controle glicêmico pela presença concomitante de hiperadrenocorticismo foi reforçada pela ultrassonografia, evidenciando aumento das glândulas adrenais, e foi confirmada através do teste de supressão por dexametasona.

O aumento bilateral das glândulas adrenais evidenciado na ultrassonografia leva à suspeita de hiperadrenocorticismo secundário à hipófise. A tomografia computadorizada e a ressonância magnética são meios diagnósticos para tumores pituitários [1], porém na época desse relato não havia tomografia computadorizada disponível na Medicina Veterinária Brasileira, não podendo confirmar a suspeita de aumento da glândula pituitária.

Sinais neurológicos como andar compulsivo e em círculo caracterizam lesões no diencéfalo e podem caracterizar uma massa na hipófise [2-4]. A gata desse relato começou a apresentar esses sinais três semanas após a cirurgia devido a adenoma hipofisário diagnosticado pela necropsia. Uma complicação da adrenalectomia bilateral é o aumento da massa 
pituitária [1], o que provavelmente aconteceu após a cirurgia e que provocou os sinais neurológicos nesse felino.

Em casos de hiperadrenocorticismo secundário a adenoma pituitário, hipofisectomia transfenoidal pode ser realizada para a retirada do adenoma [5], porém, quando isso não é possível, a adrenalectomia bilateral é indicada, fornecendo uma qualidade de vida para o paciente. A gata relatada teve uma qualidade de vida melhor após a adrenalectomia bilateral e viveu por ainda oito meses. Outra opção de tratamento para neoplasia pituitária seria a radioterapia, também não disponível para esse paciente $[3,4]$.
A adrenalectomia bilateral pode ser uma opção para tratamento de hiperadrenocorticismo secundário à neoplasia pituitária, podendo não ocorrer qualquer complicação pós-cirúrgica e dar uma qualidade de vida ao paciente melhor do que a qualidade de vida antes da cirurgia, porém, pode ocorrer aumento da neoplasia e aparecimento de sinais neurológicos no pós-operatório.

\section{NOTAS INFORMATIVAS}

${ }^{1}$ Insulina glargina, Lantus ${ }^{\circledR}$, Aventis Pharma Ltda. Grupo Sanofi-Aventis, São Paulo, Brasil.

${ }^{2}$ Dexametasona, Azium solução injetável®, Schering-

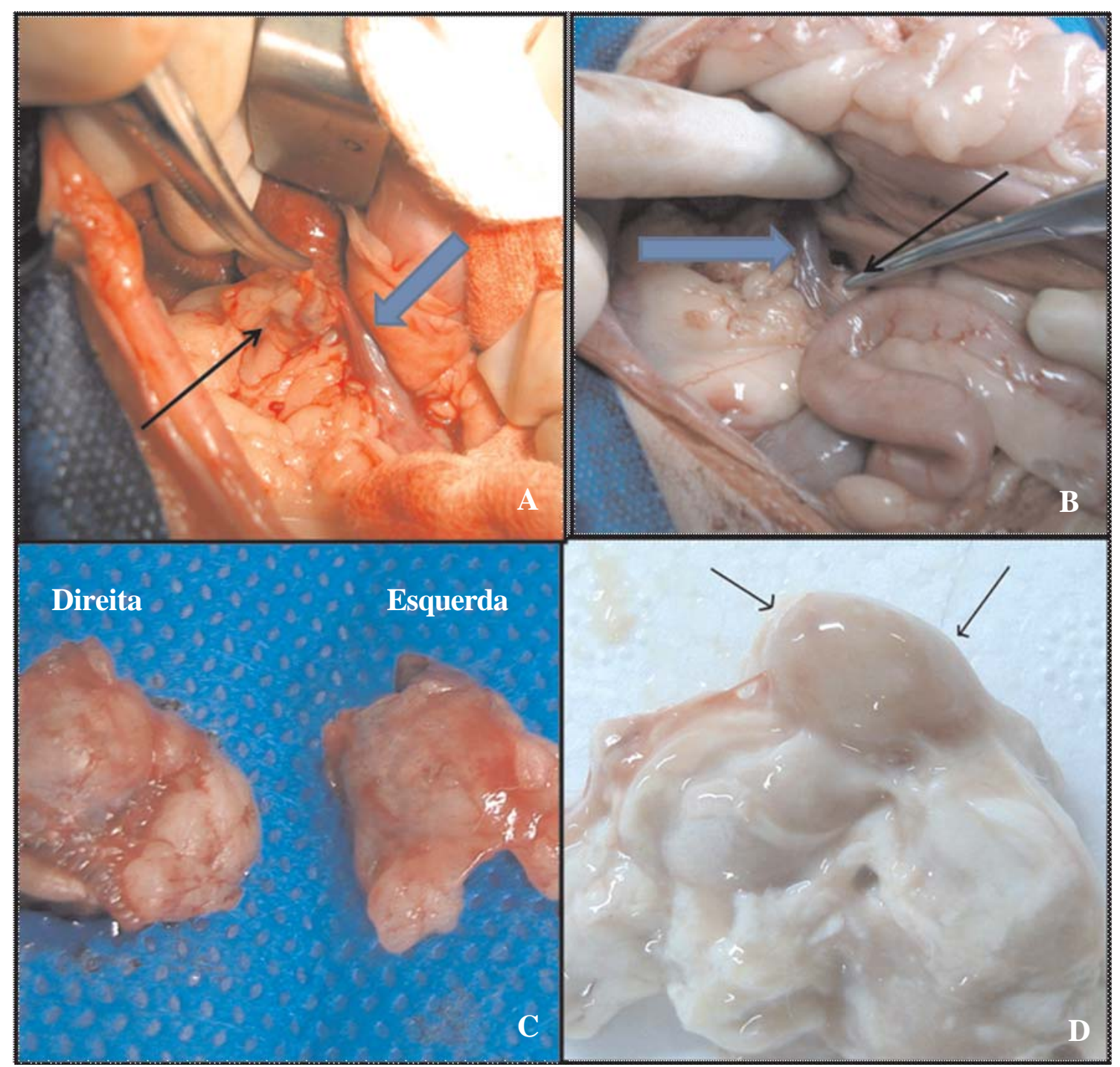

Figura 1. Gata com hiperadrenocorticismo hipofisário. A- Cirurgia de adrenalectomia. Observa-se a adrenal direita (seta preta) lateral à veia cava caudal (seta azul). B- Adrenal esquerda na ponta da pinça homeostática (seta preta e lateral à veia cava caudal (seta azul)). C- Glândulas adrenais após a retirada cirúrgica. D- Encéfalo com hipófise aumentada de tamanho (setas pretas) na necropsia da gata 8 meses após a adrenalectomia. 
Plough Veterinária-Indústria Química Farmacêutica Schering-Plough S. A., São Paulo, Brasil.

${ }^{3}$ Mitotane, Lisodren ${ }^{\circledR}$, Bristol-Myers Squibb Farmacêutica Ltda., São Paulo, Brasil.
${ }^{4}$ Fludrocortisona, Florinefe ${ }^{\circledR}$, Bristol-Myers Squibb Farmacêutica Ltda., São Paulo, Brasil.

${ }^{5}$ Prednisolona, Prelone®, Aché laboratórios Framacêuticos S/A., São Paulo, Brasil.

\section{REFERÊNCIAS}

1 Chiaramonte D. \& Greco D.S. 2007. Feline adrenal disorders. Clinical Techniques in Small Animal Practice. 22(1): 26-31. 2 Fracassi F., Mandrioli L., DianaA., Hilbe M., Grinwis G. \& Gandini G. 2007. Pituitary Macroadenoma in a Cat with Diabetes Mellitus, Hypercortisolism and Neurological Signs. Journal of Veterinary Medicine. A, Physiology, Pathology, Clinical Medicine. 54(7): 359-363.

3 Mayer M.N., Greco D.S. \& LaRue S.M. 2006. Outcomes of pituitary tumor irradiation in cats. Journal of Veterinary Internal Medicine. 20(5): 1151-1154.

4 Mayer M.N. \& Treuil P.L. 2007. Radiation therapy for pituitary tumors in the dog and cat. Canadian Veterinary Journal. 48(3): 316-318.

5 Meij B.P., Vlugt-Meijer R.H., Ingh T.S.G.A.M. \& Rijnberk A. 2004. Somatotroph and Corticotroph Pituitary Adenoma (Double Adenoma) in a Cat with Diabetes Mellitus and Hyperadrenocorticism. Journal of Comparative Pathology. 130(23): 209-215. 